\title{
Propagation of Modified Bessel-Gaussian Beams in a Misaligned Optical System
}

\author{
Lahcen Ez-Zariy $^{1}$, Hamid Nebdi ${ }^{1}$, El Hassane Bentefour ${ }^{2}$, Abdelmajid Belafhal ${ }^{1 *}$ \\ ${ }^{1}$ Laboratoire de Physique Nucléaire, Atomique et Moléculaire Département de Physique, Faculté des Sciences, \\ Université Chouaïb Doukkali, El Jadida, Morocco \\ ${ }^{2}$ LigthOnKnowledge Foundation, Chicago, USA \\ Email: "belafhal@gmail.com
}

Received August 15, 2012; revised September 15, 2012; accepted September 30, 2012

\begin{abstract}
The formalism of generalized diffraction integral for paraxial misaligned optical systems is used to investigate the propagation of the Modified Bessel-Gaussian (MBG) beam through a misaligned thin lens. The properties of the propagation of MBG beam traveling through this misaligned ABCD optical system are discussed. A special case of misaligned circular thin lens is illustrated analytically and numerically. The shape of the MBG beam at the exit of the misaligned optical system is unchanged; however the center of the beam is shifted from the propagation axis in correlated manner with the design parameters of the optical system.
\end{abstract}

Keywords: Generalized Diffraction Integral; Modified Bessel-Gaussian Beams; Propagation; Misaligned ABCD Optical Systems; Circular Thin Lens

\section{Introduction}

Modified optical beams, such as zero central intensity beams and vortex beams, gained increasing importance in the recent years [1,2]. Among their applications are the guiding and manipulating of particle beam such as in atoms interferometry, atoms trapping and cooling, and in wireless Tera-bite-speed information transport. Particular attention is given to the propagation of Bessel and Modified Bessel beams, which their apodization by a Gaussian transmittance leads to the so called, in literature, Modified Bessel-Gaussian beams (referred in the follows as MBG beams).

Several studies have examined the propagation of MBG beams through a turbulent atmosphere [3,4], and through aligned optical systems [5-11]. These studies and others [12-21] intend to comprehend practical matters such as the impact of imperfections in the optical systems on the propagation of the MBG beams. Such imperfections, which translate into misalignment of the optical systems, may be due in one hand to the design and manufacturing mishaps and in other hand due to external perturbations such as thermally induced deformations and or the accumulation of dust and fingerprints, etc. In this perspective, the propagation of nondiffracting and pseudo-nondiffracting Laser beams through misaligned optical systems is of great interest.

"Corresponding author.
Recently, Zhao et al. [17] have used the generalized diffraction integral to derive a generalized formula for high-order Bessel-Gaussian beams through a misaligned first order ABCD optical system. In 2009, Chafiq et al. [19] have applied the generalized diffraction integral to develop an analytical formalism for generalized MathieuGauss beams passing through an aperture misaligned optical system. Moreover, in a recent work, Belafhal et al. [20] have studied the propagation of truncated Bessel modulated Gaussian (QBG) beams traveling any misaligned $A B C D$ optical system using the generalized diffraction integral formalism.

To our knowledge, the propagation of MBG beams through misaligned optical systems has not been studied yet. In this paper, we use the generalized diffraction integral introduced by Wang and Ronchi [22], and all our previous work, in this field, to develop the analytical solution of the propagation of MBG beams traveling through any misaligned optical system. For illustration, we apply our solution to the particular case of the propagation of MBG beams through a misaligned lens. More interestingly, we demonstrate that our analytical result is a generated formalism which allows for retrieving the solution of the propagation of pure Bessel, Gaussian and of Bessel-Gaussian beams through a misaligned optical system [17] as particular cases.

The paper is organized as follows: in Section 2, we calculate the analytical equation of MBG beams passing 
through any misaligned optical system ABCD. Then, from this equation, we derive the solutions for the special cases of the Bessel, Gaussian and Bessel-Gaussian beams propagating through the same optical system. In Section 3 , we use the main expression of Section 2 to perform detailed analysis of the propagation of MBG through a misaligned thin lens analytically and numerically.

\section{Propagation of MBG Beams through Misaligned Paraxial Optical System}

MBG beams are solutions of the 3D Helmholtz equation in cylindrical coordinates. These beams are the generalized Modified Bessel beams modulated by Gaussian transmittance. The field distribution of MBG beams in the source plane situated at $z=0$ is composed by the product of a Gaussian function part and a summation over the modified Bessel functions of different amplitudes $A_{n}$. This field is expressed by [11]

$$
\begin{aligned}
& E\left(r_{0}, \theta_{0}, 0\right) \\
& =\exp \left(-k \alpha r_{0}^{2}\right) \sum_{n}^{N} A_{n} \exp \left(-\mathrm{i} n \theta_{0}\right) I_{n}\left(a_{B} r_{0}\right),
\end{aligned}
$$

where $n$ denotes the order of modified Bessel function $I_{n}$,

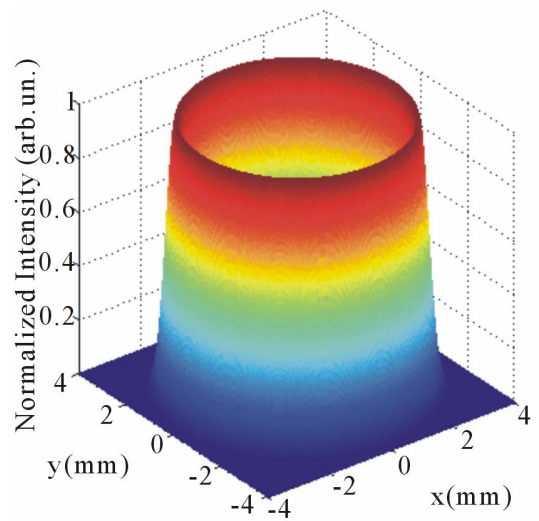

(a)

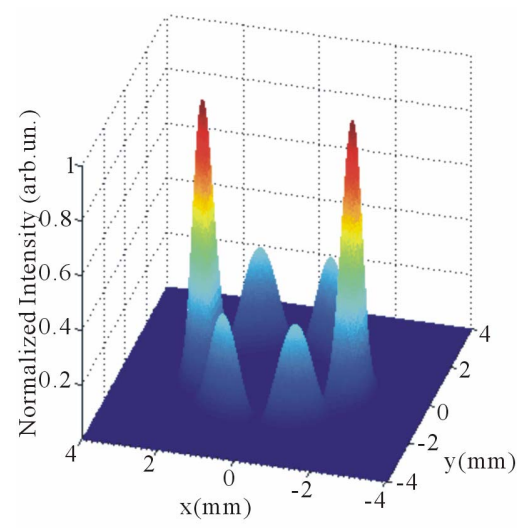

(b)
$a_{B}$ is the width parameter, $k=\frac{2 \pi}{\lambda}$ is the wave number with $\lambda$ being the wavelength, $\alpha=\frac{1}{\left(k \alpha_{s}^{2}\right)}+\frac{\mathrm{i}}{\left(2 F_{s}\right)}$, where $\alpha_{s}$ and $F_{s}$ respectively refer to radial Gaussian source and focusing parameter, $\mathrm{i}=\sqrt{(-1)}$.

In Figure 1, we present the plots of the incident single and multiple MBG beams intensity at different orders and with various amplitude coefficients. Figure 1(a) shows a single modified Bessel-Gaussian beam of order 10 which can be obtained from summation in Equation (1) by setting $n=N=10$. The diagram of the considered figure shows the single modified Bessel beam of order 10 which is a doughnut beam (this observation holds true for any single beam of order $n>0$ ). Figure 1(b) displays an example of a superposition of the modified BesselGaussian beams for different odd orders, $n$, and with various amplitudes, $A_{n}$. From this figure, it appears that the beam is a dark hollow beam, and the plot presents two principal lobes surrounded by secondary lobes. In addition, we note that the considered figure presents a
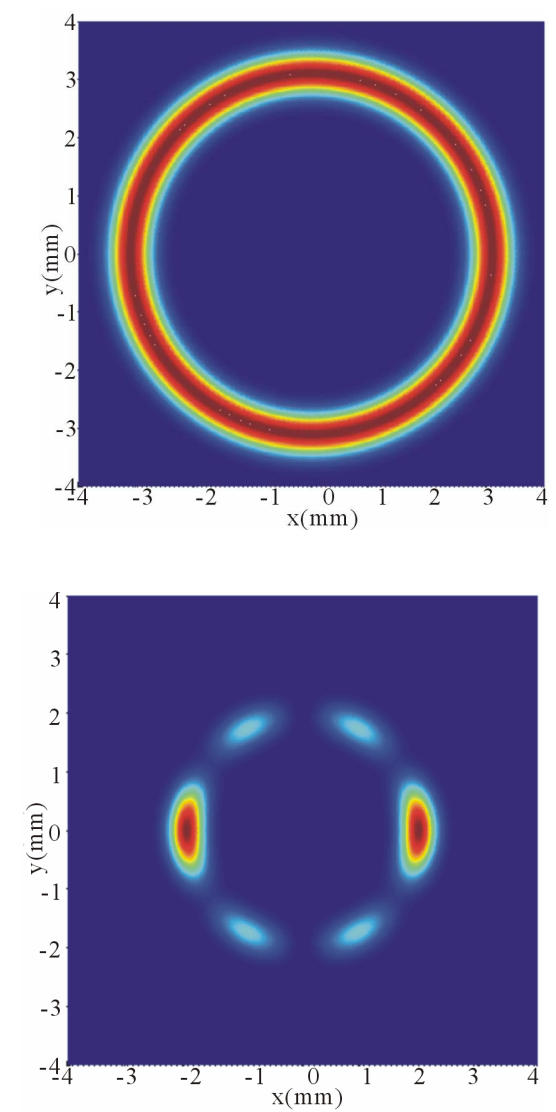

Figure 1. Normalized transverse irradiance distributions of input single and multiple MBG beams at various amplitudes: (a) $n=10 A_{n}=10 ;(\mathrm{b})\left(n=\left[\begin{array}{llll}1 & 3 & 5 & 7\end{array}\right] \quad A_{n}=\left[\begin{array}{llll}1 & 0.5 & 0.2 & 1\end{array}\right]\right)$. 


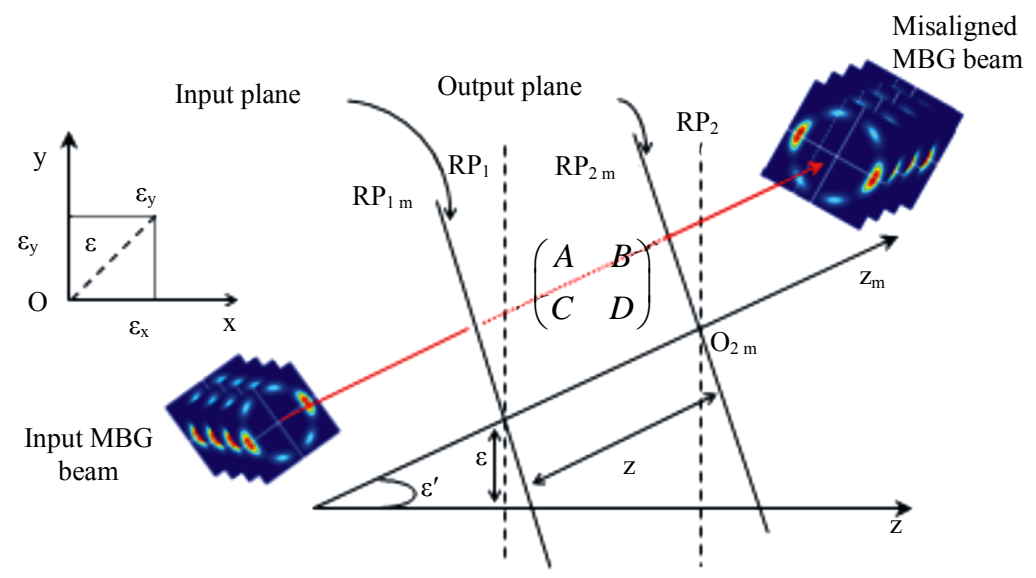

Figure 2. Schematic representation of MBG beams propagation trough a misaligned paraxial ABCD optical system.

symmetry in the lobes, because the products of $u\left(r_{0}, q_{0}\right) \cdot u *\left(r_{0}, q_{0}\right)$ generate terms defining the azimuthal intensity as $\cos \left(2 n q_{0}\right)$.

Let us now consider a misaligned ABCD optical system illuminated by a MBG beam as shown in Figure 2. $R P_{1,2}$ are the alignment reference planes, $R P_{1 \mathrm{~m}, 2 \mathrm{~m}}$ are the misalignment reference planes, $\varepsilon$ denotes the transverse offset and $\varepsilon^{\prime}$ is the tilted angle. $A, B, C$ and $D$ are the matrix transfer elements of the aligned optical system.

In a cylindrical coordinates system, the generalized diffraction integral formula between input electric field $E_{0}\left(r_{0}, \theta_{0}, z=0\right)$ and output plane electric field $E(r, \theta, z)$ is given by [17]

$$
\begin{aligned}
& E(r, \theta, z)=\frac{\mathrm{i} k}{2 \pi B} \exp (-\mathrm{i} k z) \int_{0}^{2 \pi} \int_{0}^{+\infty} E\left(r_{0}, \theta_{0}, z=0\right) \\
& \times \exp \left\{-\frac{\mathrm{i} k}{2 B}\left[A r_{0}^{2}-2 r r_{0} \cos \left(\theta-\theta_{0}\right)+D r^{2}+E r_{0} \cos \theta_{0}\right.\right. \\
& \left.\left.+F r_{0} \sin \theta_{0}+G r \cos \theta+H r \sin \theta\right]\right\} r_{0} \mathrm{~d} r_{0} \mathrm{~d} \theta_{0}
\end{aligned}
$$

The misalignment parameters $E, F, G$, and $H$ characterizing the optical system are given by

$$
\begin{aligned}
E & =2\left[(1-A) \varepsilon_{x}+(z-B) \varepsilon_{x}^{\prime}\right], \\
F & =2\left[(1-A) \varepsilon_{y}+(z-B) \varepsilon_{y}^{\prime}\right], \\
G= & -2[B C+D(1-A)] \varepsilon_{x} \\
& -2[B(1-D)+D(z-B)] \varepsilon_{x}^{\prime}, \\
H= & -2[B C+D(1-A)] \varepsilon_{y} \\
& -2[B(1-D)+D(z-B)] \varepsilon_{y}^{\prime}
\end{aligned}
$$

where $\varepsilon_{x}, \varepsilon_{x}^{\prime}, \varepsilon_{y}$ and $\varepsilon_{y}^{\prime}$ denote the two-dimensional misalignment parameters. $\varepsilon_{x}$ and $\varepsilon_{y}$ are the displacement element in $x$ and $y$ direction, respectively. $\varepsilon_{x}^{\prime}$ and $\varepsilon_{y}^{\prime}$ are the tilting angle of the element in $x$ and $y$ direction, respectively. The parameters $\varepsilon_{x}$ and $\varepsilon_{y}$ are given by

$$
\varepsilon_{x}=\varepsilon \cos \varphi,
$$

and

$$
\varepsilon_{y}=\varepsilon \sin \varphi .
$$

Here $\varphi$ is the misaligned azimuth angle of the optical elements in a cylindrical coordinates system. Also $\varepsilon_{x}^{\prime}$ and $\varepsilon_{y}^{\prime}$ are given by

$$
\varepsilon_{y}=\varepsilon \sin \varepsilon_{x}^{\prime}=\arctan \left(\cos \varphi \tan \varepsilon^{\prime}\right),
$$

and

$$
\varepsilon_{x}^{\prime}=\arctan \left(\sin \varphi \tan \varepsilon^{\prime}\right) .
$$

Substituting Equation (1) into Equation (2a), we get

$$
\begin{aligned}
& E(r, \theta, z) \\
& =\frac{i k}{2 \pi B} \mathrm{e}^{-\mathrm{i} k z} \exp \left\{-\frac{\mathrm{i} k}{2 B}\left[D r^{2}+G r \cos \theta+H r \sin \theta\right]\right\} \\
& \sum_{n}^{N} A_{n} \int_{0}^{+\infty} \exp \left\{-\left(\frac{\mathrm{i} k}{2 B} A+k \alpha\right) r_{0}^{2}\right\} I_{n}\left(a_{B} r_{0}\right) \\
& \times\left(\int _ { 0 } ^ { 2 \pi } \operatorname { e x p } ( - \mathrm { in } \theta _ { 0 } ) \operatorname { e x p } \left\{-\frac{\mathrm{i} k}{2 B}\left[\left(-2 r r_{0} \cos \left(\theta-\theta_{0}\right)\right)\right.\right.\right. \\
& \left.\left.\left.+E r_{0} \cos \theta_{0}+F r_{0} \sin \theta_{0}\right]\right\} \mathrm{~d} \theta_{0}\right) r_{0} \mathrm{~d} r_{0} .
\end{aligned}
$$

For solving the double integral of Equation (3), one introduce a new parametric angle $\phi$ and a new radial coordinate $\rho$ which satisfy the following relations

$$
\begin{aligned}
& \cos \phi=\frac{r \cos (\theta)-E / 2}{\sqrt{(r \cos (\theta)-E / 2)^{2}+(r \sin (\theta)-F / 2)^{2}}}, \\
& \sin \phi=\frac{r \sin (\theta)-F / 2}{\sqrt{(r \cos (\theta)-E / 2)^{2}+(r \sin (\theta)-F / 2)^{2}}},
\end{aligned}
$$

and 


$$
\rho=\sqrt{(r \cos (\theta)-E / 2)^{2}+(r \sin (\theta)-F / 2)^{2}} .
$$

After a tedious calculations and with help of the below integral formulae [23],

$$
\begin{aligned}
& \int_{0}^{2 \pi} \exp [\mathrm{i} x \cos (\xi-\psi)-\mathrm{im} \psi] \mathrm{d} \psi \\
& =2 \pi \mathrm{i}^{m} \exp (-\mathrm{im} \xi) J_{m}(x)
\end{aligned}
$$

and

$$
\int_{0}^{\infty} x \mathrm{e}^{-\delta x^{2}} I_{v}(\beta x) \times J_{v}(\gamma x) \mathrm{d} x=\frac{1}{2 \alpha} \exp \left(\frac{\beta^{2}-\gamma^{2}}{4 \delta}\right) J_{v}\left(\frac{\beta \gamma}{2 \delta}\right) \text { with }[\operatorname{Re} \delta>0, \quad \operatorname{Re} v>-1],
$$

the receiver electric field of a MBG beam passing by any misaligned ABCD optical system, is expressed by

$$
\begin{aligned}
E(r, \theta, z)= & \frac{\mathrm{i} k}{(2 k \alpha B+\mathrm{i} k A)} \mathrm{e}^{-\mathrm{i} k z} \exp \left\{-\frac{\mathrm{i} k}{2 B}\left[D r^{2}+G r \cos \theta+H r \sin \theta\right]\right\} \\
& \times \frac{1}{2 \delta} \exp \left(\frac{\beta^{2}-\gamma^{2}}{4 \delta}\right) \times \sum_{n}^{N} A_{n} \exp \left(\operatorname{in}\left(\frac{\pi}{2}-\phi\right)\right) \times J_{n}\left(\frac{\beta \gamma}{2 \delta}\right),
\end{aligned}
$$

where

$$
\begin{aligned}
\delta=k \alpha+\mathrm{i} \frac{k A}{2 B}, & (7 \mathrm{a}) \quad \begin{array}{c}
\text { Taking in account the expression } \\
\text { can be written as }
\end{array} \\
\gamma=\frac{k \rho}{B}, & \\
E(r, \theta, z)= & \frac{\mathrm{i} k}{(2 k \alpha B+\mathrm{i} k A)} \mathrm{e}^{-\mathrm{i} k z} \exp \left\{-\frac{\mathrm{i} k}{2 B}\left[D r^{2}+G r \cos (\theta)+H r \sin (\theta)\right]\right\} \\
& \times \exp \left[\frac{a_{B}^{2}}{4\left(k \alpha+\mathrm{i} \frac{k A}{2 B}\right)}-\frac{k^{2}\left((2 r \cos (\theta)-E)^{2}+(2 r \sin (\theta)-F)^{2}\right)}{16 B^{2}\left(k \alpha+\mathrm{i} \frac{k A}{2 B}\right)}\right] \\
& \times \sum_{n}^{N} A_{n} \exp \left(\mathrm{in}\left(\frac{\pi}{2}-\phi\right)\right) \times J_{n}\left(\frac{k a_{B} \sqrt{(2 r \cos \theta-E)^{2}+(2 r \sin \theta-F)^{2}}}{4 B\left(k \alpha+\mathrm{i} \frac{k A}{2 B}\right)}\right) .
\end{aligned}
$$

and

Taking in account the expression of $\rho$, Equation (6)

This formula is the general analytical equation of output electric field of a MBG beam traveling a misaligned paraxial ABCD optical system and it's the main result of this paper. From Equations (4c) and (8), we can easily deduce that the beam obtained at the misaligned plane after the optical system becomes decentered. The position of the center of the output beam is shifted from the center of emitted plane beam by $\mathrm{E} / 2$ in $\mathrm{x}$-direction and by $F / 2$ in $y$-direction.

The validity of the present work withstands the generalized formalism obtained in the previous investigations about propagating of pure Bessel beams, pure Gaussian beams and Bessel-Gaussian beams through a misaligned optical system [20].

Taking in account the following variables changing

$$
N=n \text {, }
$$

$$
\begin{aligned}
& A_{n}(-\mathrm{i})^{n}=E_{0}, \\
& \mathrm{i} a_{B}=\alpha_{Z}, \\
& k \alpha=\frac{1}{w_{\circ}^{2}},
\end{aligned}
$$

Equation (1) becomes

$$
\begin{aligned}
& E\left(r_{0}, \theta_{0}, 0\right) \\
& =E_{0} J_{n}\left(\alpha_{Z} r_{0}\right) \exp \left(-\frac{r_{0}^{2}}{w_{0}^{2}}\right) \exp \left(-\mathrm{in} \theta_{0}\right),
\end{aligned}
$$

that is the high order Bessel-Gaussian beam which is the incident electric field expressed by Equation (7) of Ref. [17]. By substituting Equations (9) in Equation (8) of our investigation, we obtain 


$$
\begin{aligned}
E_{B G}(r, \theta, z)= & \frac{\mathrm{ik} E_{0}}{2 B\left(\frac{1}{w_{0}^{2}}+\mathrm{i} \frac{k A}{2 B}\right)} \mathrm{e}^{-\mathrm{i} k z} \exp \left\{-\frac{\mathrm{i} k}{2 B}\left[D r^{2}+G r \cos \theta+H r \sin \theta\right]\right\} \exp (-\mathrm{in} \phi) \\
& \times \exp \left\{-\frac{\alpha_{z}^{2}}{4\left(\frac{1}{w_{0}^{2}}+\mathrm{i} \frac{k A}{2 B}\right)}-\frac{k^{2}\left[(2 r \cos \theta-E)^{2}+(2 r \sin \theta-F)^{2}\right]}{16 B^{2}\left(\frac{1}{w_{0}^{2}}+\mathrm{i} \frac{k A}{2 B}\right)}\right\} \\
& \times J_{n}\left(\frac{\mathrm{i} k \alpha_{z} \sqrt{(2 r \cos \theta-E)^{2}+(2 r \sin \theta-F)^{2}}}{4 B\left(\frac{1}{w_{0}^{2}}+\mathrm{i} \frac{k A}{2 B}\right)}\right)
\end{aligned}
$$

This result describes the Bessel-Gaussian beam after passing through a misaligned paraxial optical system that is the main finding of Zhao et al. [17]. When $w_{0} \rightarrow \infty$, this equation can be reduced to the propagation equation through a misaligned paraxial optical ABCD system of a pure Bessel beam, which is given by

$$
\begin{aligned}
E_{B}(r, \theta, z)= & \frac{E_{0}}{A} \mathrm{e}^{-\mathrm{i}(k z+n \phi)} \exp \left\{-\frac{\mathrm{i} k}{2 B}\left[D r^{2}+G r \cos \theta+H r \sin \theta\right]\right\} \\
& \times \exp \left\{\frac{\mathrm{i}}{2 A}\left[\frac{\alpha_{Z} B}{k}+\frac{k}{4 B} \cdot\left((2 r \cos \theta-E)^{2}+(2 r \sin \theta-F)^{2}\right)\right]\right\} \\
& \times J_{n}\left(\frac{\alpha_{Z}}{2 A} \sqrt{(2 r \cos \theta-E)^{2}+(2 r \sin \theta-F)^{2}}\right) .
\end{aligned}
$$

The Gaussian case corresponds to $\alpha=0$ and $n=0$, so the corresponding output electric field is given by

$$
\begin{aligned}
E_{G}(r, \theta, z)= & \frac{\mathrm{i} k E_{0}}{2 B\left(\frac{1}{w_{0}^{2}}+\mathrm{i} \frac{k A}{2 B}\right)} \mathrm{e}^{-\mathrm{i} k z} \exp \left\{-\frac{\mathrm{i} k}{2 B}\left[D r^{2}+G r \cos \theta+H r \sin \theta\right]\right\} \\
& \times \exp \left\{-\frac{k^{2}}{16 B^{2}\left(\frac{1}{w_{0}^{2}}+\mathrm{i} \frac{k A}{2 B}\right)}\left((2 r \cos \theta-E)^{2}+(2 r \sin \theta-F)^{2}\right)\right\} .
\end{aligned}
$$

Next, we will be interested to the normalized intensity of the receiver beam which is given by $I_{N}=E(r, \theta, z) \times E^{*}(r, \theta, z) / I_{\max }$, where * denotes the conjugate and $I_{\max }$ is the maximal intensity.

\section{Numerical Example: Propagation of MBG Beams through a Misaligned Thin Lens}

In order to validate our calculation of the propagation of MBG beams through a misaligned optical system, we studied the propagation of the considered beam through a circular thin lens.

The displacements and angle misalignments of the lens with respect to the optical axis of the system are respec- tively: $\varepsilon_{x} \neq 0$ and $\varepsilon_{y}=\varepsilon_{x}^{\prime}=\varepsilon_{x}^{\prime}=0$ (see Figure (3)).

The thin lens is located at $z=0$ and the exit plane is located at $z$. The ray transfer matrix of the optical system between the input plane and the exit plane are

$$
\left(\begin{array}{ll}
A & B \\
C & D
\end{array}\right)=\left(\begin{array}{ll}
1-z / f & z \\
-1 / f & 1
\end{array}\right),
$$

and from Equations (2), one deduces

$$
E=2 \frac{z \varepsilon_{x}}{f} \text { and } F=G=H=0 .
$$

where $f$ is the focal length of the thin lens. 


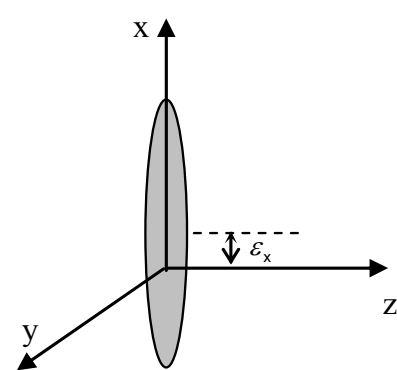

(a)

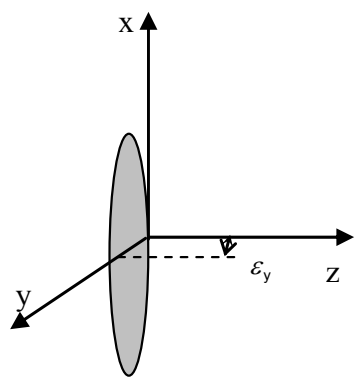

(b)

Figure 3. Misaligned thin lens: (a) displacement $\varepsilon_{x}$ in $\mathrm{x}$-direction, (b) displacement $\varepsilon_{y}$ in y-direction.

In this case, Equation (4c) becomes $\rho=\sqrt{(x-E / 2)^{2}+y^{2}}$. From this, one can deduce that the beam center in exit plane is deviated from the optical axis only in x-direction by $E / 2=z \varepsilon_{x} / f$.

For another use of a defined lens, we choose $\varepsilon_{y} \neq 0$ and $\varepsilon_{x}=\varepsilon_{x}^{\prime}=\varepsilon_{x}^{\prime}=0$. In this case, we have

$$
F=2 \frac{z \varepsilon_{y}}{f} \text { and } E=G=H=0 .
$$

Similarly in this case, one obtains
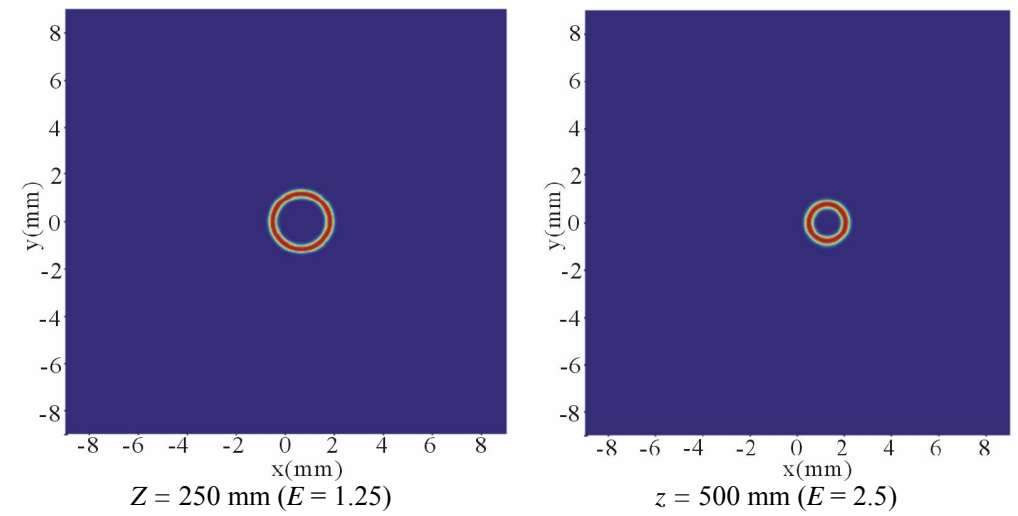

$z=500 \mathrm{~mm}(E=2.5)$

(a)

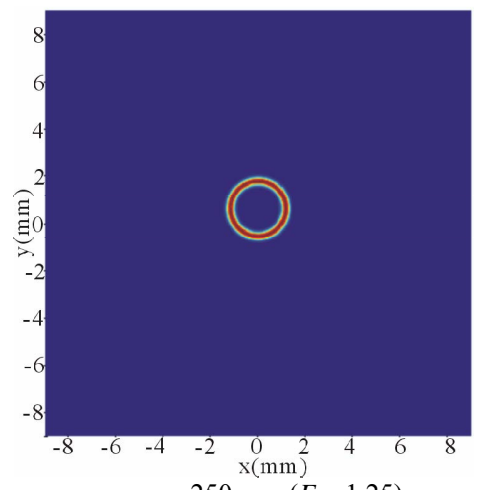

$\mathrm{z}=250 \mathrm{~mm}(F=1.25)$

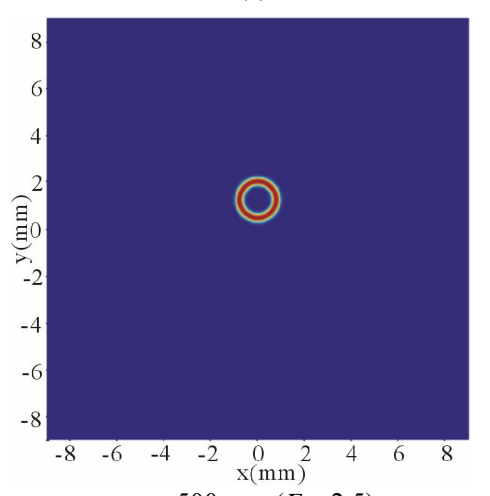

$z=500 \mathrm{~mm}(F=2.5)$

(b)
$\rho=\sqrt{x^{2}+(y-F / 2)^{2}}$ and the center beam will be shifted only in y-direction by $F / 2=z \varepsilon_{y} / f$.

To confirm our theoretical finding numerically, we illustrate in Figures $\mathbf{4}$ and $\mathbf{5}$ the analytical results related to the propagation of MBG beam through the considered misaligned thin lens. In the numerical calculations, the parameters of the beam and of the optical system chosen are: $\lambda=0.6328 \mu \mathrm{m}$, the width parameter $a_{B}$ is fixed at $a_{B}=24.810 \mathrm{~mm}^{-1}$, the radial Gaussian size is $a_{\mathrm{s}}=1 \mathrm{~cm}$, and we choose that the focusing parameter $\mathrm{F}_{\mathrm{s}} \rightarrow \infty$. For all figures, we present the normalized intensity distributions of the single and multiple MBG beams of various orders. At $z=0$, before propagation through the misaligned lens, the center of the beam is located on the optical axis. However, in the case of displacement of the optical system in $\mathrm{x}$-direction by $\varepsilon_{x}=1 \mathrm{~mm} \quad$ (see Figure 3(a)) and taking

$\varepsilon_{y}=\varepsilon_{x}^{\prime}=\varepsilon_{y}^{\prime}=0$, from the curves of Figures 4(a) and 5(a), it can be seen that the center of the existing beam effectively is decentred in $\mathrm{x}$-direction. The corresponding displacements for the propagation distances $z=0.25 ; 0.5$; 1 and $1.5 \mathrm{~m}$ are respectively: $\frac{E}{2} \approx 0.625 ; 1.25$ and 2.5 .

Figure 4. Contour maps of normalized three-dimensional intensity distributions of the output single MBG beam of order $n=$ 10 through a misaligned thin lens at various $z$. Displacement of optical system by: (a) $\varepsilon_{x}=1 \mathrm{~mm}$ in $\mathrm{x}$-direction, (b) $\varepsilon_{y}=1 \mathrm{~mm}$ in y-direction. 

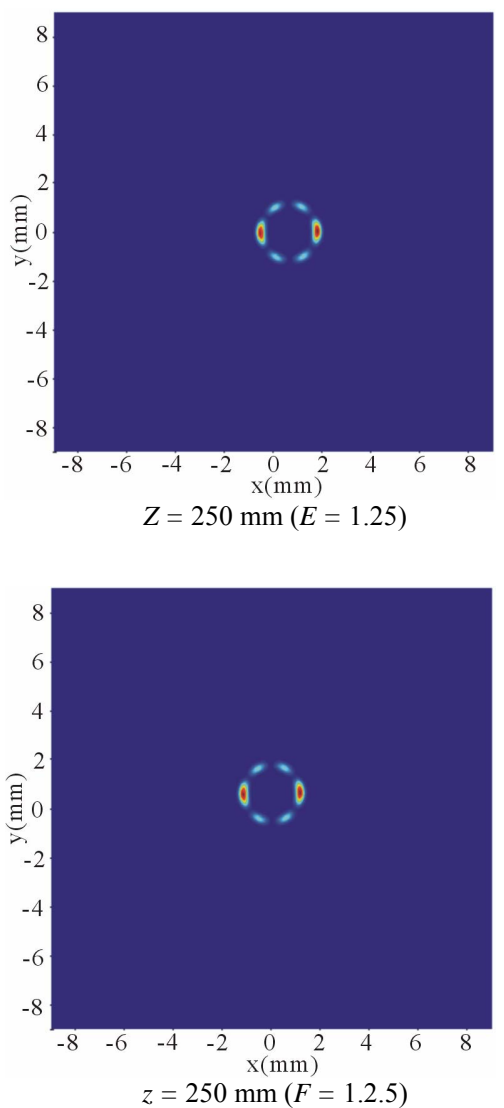

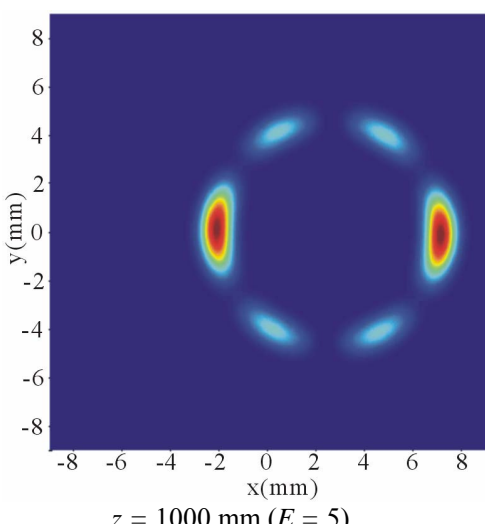

(a)

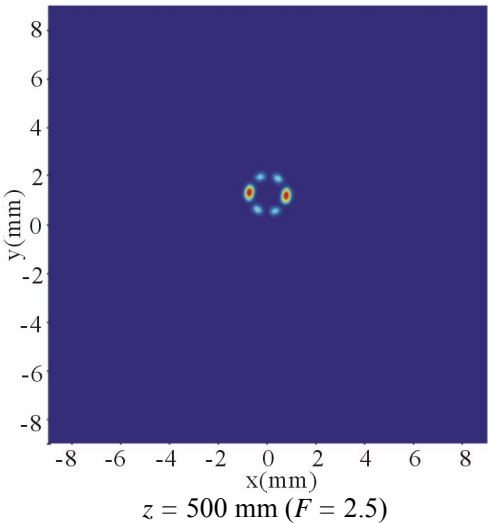

(b)

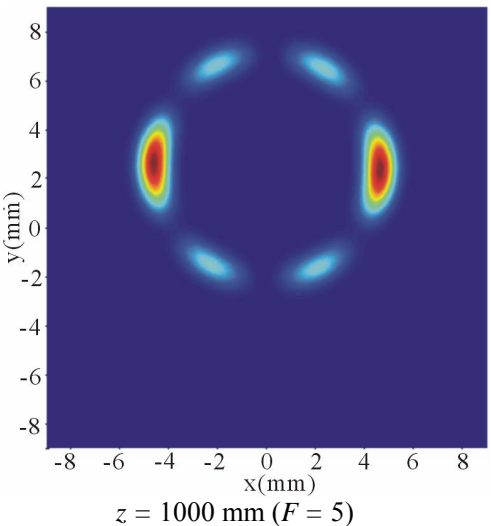

Figure 5. Contour maps of normalized three-dimensional intensity distributions of the output multiple MBG beams at odd orders $\left(n=\left[\begin{array}{llll}1 & 3 & 5 & 7\end{array}\right] A_{n}=\left[\begin{array}{llll}1 & 0.5 & 0.2 & 1\end{array}\right]\right)$ through a misaligned thin lens at various $z$. Displacement of optical system by: (a) $\varepsilon_{x}$ $=1 \mathrm{~mm}$ in $\mathrm{x}$-direction, (b) $\varepsilon_{y}=1 \mathrm{~mm}$ in y-direction.

In the same way, for the case of a displacement of optical system in y-direction by a value of $\varepsilon_{y}=1 \mathrm{~mm}$ (see Figure 3(b)) and if we take $\varepsilon_{x}=\varepsilon_{x}^{\prime}=\varepsilon_{y}^{\prime}=0$, the Figures 4(b) and 5(b) show that the normalized intensity center at receiver plane is shifted in $y$-direction. The new center is located at $(0 ; F / 2 ; z)$. The deviation angle depends on the misalignment parameters and the propagation distance $z$.

The MBG beams passing through a misaligned optical system has the same properties of the other beams in the same situation.

\section{Conclusion}

Based on the generalized diffraction integral formalism, a convenient analytical solution to the propagation of the MBG beam throughany misaligned optical system is derived. The solutions for the propagation of pure Bessel, Gaussian and Bessel-Gaussian beams, traveling through the same misaligned optical system can be deduced as special cases using our result. For the purpose of illustration, the propagation properties of the MBG beam through a misaligned thin lens were addressed as numerical example. The analysis of the result showed that the MBG beam becomes decentered after passing through a misaligned thin lens and the centeroid of the intensity distribution is displaced in dependence of the optical system misalignment parameters.

\section{REFERENCES}

[1] J. Yin, Y. Zhu, W. Jhe and Y. Wang, "Atom Guiding and Cooling in a Dark Hollow Laser Beam," Physical Review A, Vol. 58, No. 1, 1998, pp. 509-513. doi:/10.1103/PhysRevA.58.509

[2] J. P. Yin, W. J. Gao, H. F. Wang, Q. Long and H. Z. Wang, "Generations of Dark Hollow Beams and Their Applications in Laser Cooling of Atoms and All Optical-Type Bose-Einstein Condensation," Chinese Physics, Vol. 11, No. 11, 2002, pp. 1157-1169. doi:/10.1088/1009-1963/11/11/312

[3] H. T. Eyyuboglu and F. Hardalaç, "Propagation of Modified Bessel-Gaussian Beams in Turbulence," Optics \& Laser Technology, Vol. 40, No. 2, 2008, pp. 343-351. doi:/10.1016/j.optlastec.2007.06.006 
[4] H. T. Eyyuboglu, Y. Baykal, E. Sermutlu, O. Korotkova and Y. Cai, "Scintillation Index of Modified BesselGaussian Beams Propagating in Turbulent Media," Journal of the Optical Society of America, Vol. 26, No. 2, 2009, pp. 387-394. doi:/10.1364/JOSAA.26.000387

[5] S. A. Ponomarenko, "A Class of Partially Coherent Beams Carrying Optical Vortices," Journal of the Optical Society of America, Vol. 18, No. 1, 2001, pp. 150-156. doi:/10.1364/JOSAA.18.000150

[6] L. Wang, X. Wang and B. Lü, "Propagation Properties of Partially Coherent Modified Bessel-Gauss Beams," Optik, Vol. 116, No. 2, 2005, pp. 65-70. doi:/10.1016/j.ijleo.2004.11.006

[7] Z. H. Gao and B. D. Lü, "Partially Coherent Nonparaxial Modified Bessel-Gauss Beams," Chinese Physics, Vol. 15, No. 2, 2006, pp. 334-339. doi:/10.1088/1009-1963/15/2/018

[8] L. Wang, M. Li, X. Wang and Z. Zhang, "Focal Switching of Partially Coherent Modified Bessel-Gaussian Beams Passing through an Astigmatic Lens with Circular Aperture," Optics \& Laser Technology, Vol. 41, No. 5, 2009, pp. 586-589. doi:/10.1016/j.optlastec.2008.10.008

[9] K. C. Zhu, X. Y. Li, X. J. Zheng and H. Q. Tang, "Nonparaxial Propagation of Linearly Polarized Modified Bessel-Gaussian Beams and Phase Singularities of the Electromagnetic Field Components," Applied Physics B: Lasers and Optics, Vol. 98, No. 2-3, 2010, pp. 567-572. doi:/10.1007/s00340-009-3807-2

[10] C. Ding, L. Pan and B. Lü, "Changes in the State of Polarization of Apertured Stochastic Electromagnetic Modified Bessel-Gauss Beams in Free-Space Propagation," Applied Physics B: Lasers and Optics, Vol. 99, No. 1-2, 2010, pp. 307-315. doi:/10.1007/s00340-009-3818-Z

[11] A. A. A. Ebrahim, L. Ez-zariy and A. Belafhal, "Propagation of Modified Bessel-Gaussian Beams through an Annular Apertured Paraxial ABCD Optical System," Physical and Chemical News, Vol. 61, 2011, pp. 52-58.

[12] G. Ding and B. Lu, "Decentered Twisted Gaussian SchellModel Beams and Their Propagation through a Misaligned First-Order Optical System," Optical and Quantum Electronics, Vol. 35, No. 2, 2003, pp. 91-100. doi:/10.1023/A:1022478608090

[13] M. Shen, S. Wang and D. Zhao, "Propagation of Flattened Gaussian Beams Passing through a Misaligned Op- tical System with Finite Aperture," Optik, Vol. 115, No. 5, 2004, pp. 193-196. doi:/10.1078/0030-4026-00346

[14] J. Gu, D. Zhao and Z. Mei, "The Relative Phase Shift of Off-Axial Gaussian Beams through an Apertured and Misaligned Optical System," Optik, Vol. 115, No. 4, 2004, pp. 187-191. doi:/10.1016/S0030-4026(08)70009-3

[15] Y. Cai and L. Zhang, "Propagation of a Hollow Gaussian Beam through a Paraxial Misaligned Optical System," Optics Communications, Vol. 265, No. 2, 2006, pp. $607-$ 615. doi:/10.1016/j.optcom.2006.03.070

[16] Y. Cai and X. Lu, "Propagation of Bessel and BesselGaussian Beams through an Unapertured or apertured Misaligned Paraxial Optical Systems," Optics Communications, Vol. 274, No. 1, 2007, pp. 1-7. doi:/10.1016/j.optcom.2007.01.058

[17] C. Zhao, L. Wang, X. Lu and H. Chen, "Propagation of High-Order Bessel-Gaussian Beam through a Misaligned First-Order Optical System," Optics \& Laser Technology, Vol. 39, No. 6, 2007, pp. 1199-1203. doi:/10.1016/j.optlastec.2006.08.015

[18] H. T. Eyyuboglu, "Propagation Aspects of MathieuGaussian Beams in Turbulence," Applied Physics B: Lasers and Optics, Vol. 91, No. 3-4, 2008, pp. 629-637. doi:/10.1007/s00340-008-3020-8

[19] A. Chafiq, Z. Hricha and A. Belafhal, "Propagation of Generalized Mathieu-Gauss Beams through Paraxial Misaligned Optical Systems," Optics Communications, Vol. 282, No. 19, 2009, pp. 3934-3939. doi:/10.1016/j.optcom.2009.03.062

[20] A. Belafhal, M. Yaalou and S. Hennani, "Propagation of Bessel-Modulated Gaussian Beams through a Misaligned First-Order Optical System," Physical and Chemical News, Vol. 61, 2011, pp. 34-43.

[21] A. Belafhal and S. Hennani, "A Note on Some Integrals Used in Laser Field Involving the Product of Bessel Functions," Physical and Chemical News, Vol. 61, 2011, pp. 59-62.

[22] S. Wang and L. Ronchi, "III Principles and Design of Optical Arrays," Progress in Optics, Vol. 25, 1988, pp. 279-348.

[23] I. S. Gradshteyn and I. M. Ryzhik, "Tables of Integrals, Series, and Products," 5th Edition, Academic Press, New York, 1994. 